\title{
rensersurnsion
}

\section{Graft surveillance in small bowel transplantation}

A study published in Transplantation has confirmed that biopsies should be performed as part of graft surveillance in patients who undergo small bowel transplantation.

An important advantage of small bowel graft surveillance is that rejection can actually be visualized by endoscopy. Stephen O'Keefe and colleagues conducted a study to investigate the accuracy of endoscopic visualization versus biopsy in the detection of acute cellular rejection of small bowel grafts. Endoscopies and biopsies were performed in 66 asymptomatic and 71 symptomatic small bowel transplant recipients from a single centre.

The sensitivity and specificity of endoscopic visualization for detecting acute cellular rejection were $50 \%$ and $91.5 \%$ for the asymptomatic patients and $43 \%$ and $67 \%$ for the symptomatic patients (compared with 100\% for biopsy). In symptomatic patients, endoscopic evaluation detected all cases of severe rejection, but 20 cases of moderate rejection were missed. In a subset of endoscopies, zoom (magnifying) endoscopy did not improve the detection of rejection.

"Endoscopic visualization of the intestinal graft is not good enough to detect early rejection," says O'Keefe. “This finding is important as reversal of early rejection is easy, whilst late rejection can result in loss of the graft." Thus, biopsy is mandatory in this setting.

The researchers conclude that the development of novel noninvasive techniques, such as confocal endoscopy, is needed to detect early rejection in small bowel transplantation.

Isobel Franks

Original article O'Keefe, S. J. et al. Endoscopic evaluation of small intestine transplant grafts. Transplantation doi:10.1097/TP.0b013e31825f4410 